\title{
Peritoneal carcinomatosis secondary to spread of hepatocellular carcinoma originated upon a healthy liver
}

\author{
Alicia Mesa ${ }^{1}$, Amador Prieto $^{1}$, Nelson Fuentes-Martínez ${ }^{2}$, Lorena Blanco-García ${ }^{3}$, \\ Olegario Castaño-Fernández ${ }^{3}$, Carmen García-Bernardo ${ }^{4}$ and María Varela ${ }^{3}$
}

Departments of ${ }^{1}$ Radiodiagniosis, ${ }^{2}$ Pathology, ${ }^{3}$ Gastroenterology and Hepatology, and ${ }^{4}$ General Surgery. Hospital Universitario Central de Asturias. Oviedo, Asturias. Spain

\section{CASE REPORT}

This is a female, 34 years, who presented with pain at the right iliac fosse and pre-syncopal symptoms. As background, she has undergone a right ovarian cystectomy due to a serous cystadenoma several years ago and appendectomy. At physical examination she had signs of peritoneal irritation and heterogeneous free fluid was detected on ultrasound, suggesting hemoperitoneum, so urgent laparotomy was decided. A peripheral liver tumor was seen in segment VII, it was partially removed and pathological diagnosis was hepatocellular carcinoma (HCC). At that time, liver function tests and alpha-fetoprotein were within normal limits and hepatitis $\mathrm{B}$ and $\mathrm{C}$ virus serology were negative. The patient was moved to a referral centre, where a triphasic liver CT and MR-angiography (Fig. 1) were performed. Small remaining tumor was observed within segment VII of the liver, without distance disease, so a formal resection of segment VII was made. The specimen showed a grade II HCC, $20 \mathrm{~mm}$ of diameter, with a satellite $1 \mathrm{~mm}$ away, without vascular invasion (Fig. 3A). No systemic or local therapy as secondary prophylaxis was administered. The fourth-month follow-up control showed multiple peritoneal implants

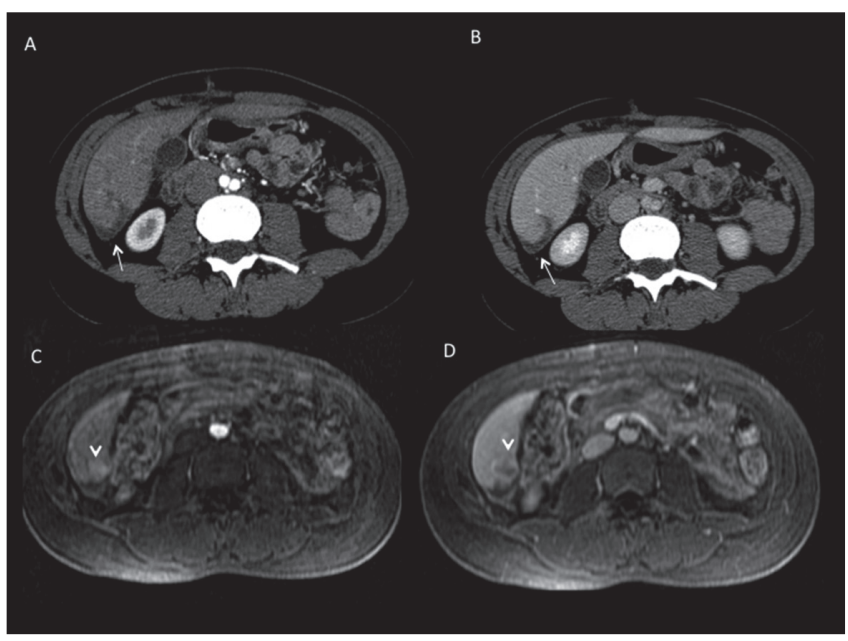

Fig. 1. A and B. Dynamic MDCT arterial and portal phase. Remaining tumor is identified (arrow) with a very dim enhancement in the arterial phase and washing up in the portal phase. C and D. Dynamic MRI. It confirms the remaining HCC with enhancement in the arterial phase, washing up in the portal phase and capsular enhancement.

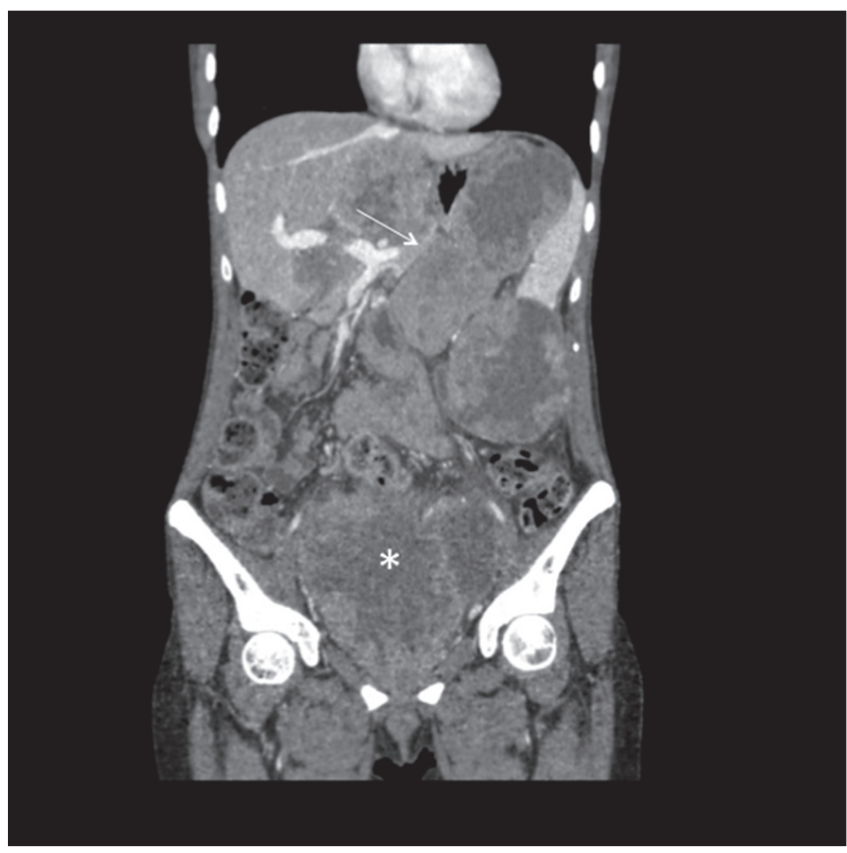

Fig. 2. MDCT with intravenous contrast, coronal plane. Numerous implants particularly striking in gastrosplenic ligament (arrow) and pelvis (asterisk) are shown. 


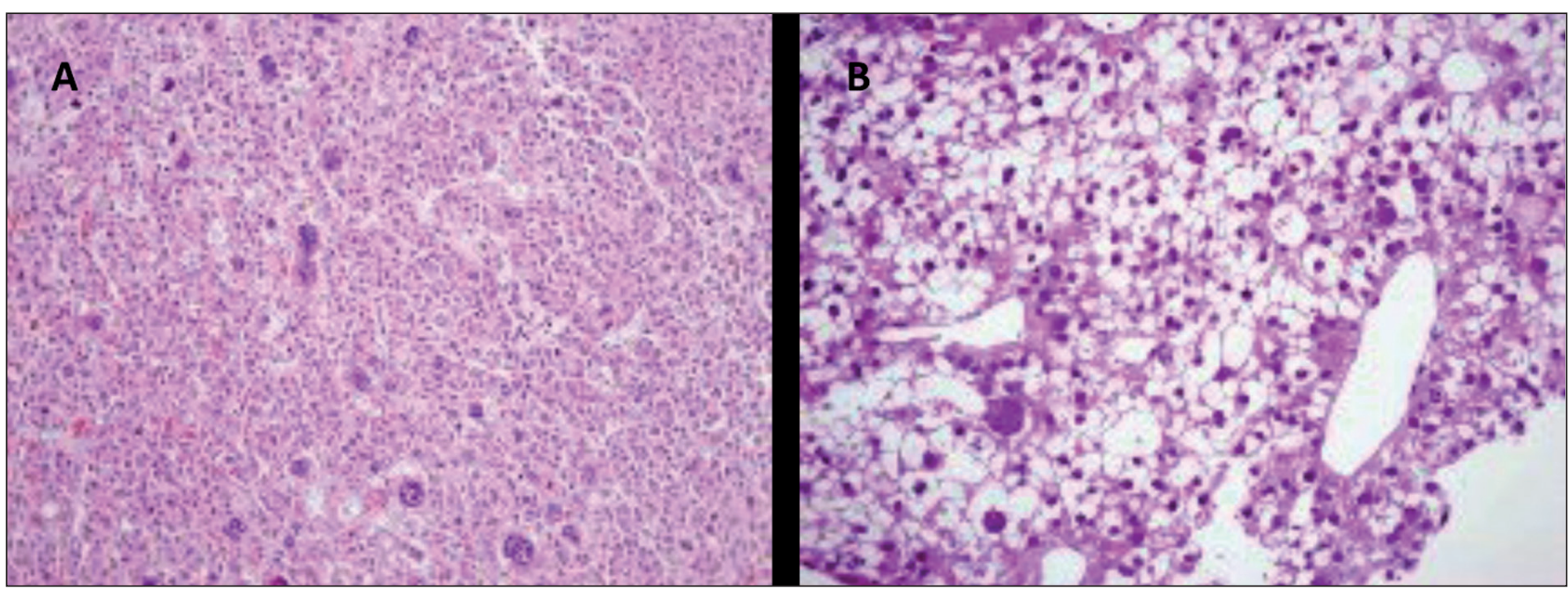

Fig. 3. A. An HCC with trabecular pattern is detected in the histological section of the surgical specimen. Pleomorphic cells with clear cytoplasm arranged in thick trabeculae over 5 hepatocytes, some bizarre, can be observed. HE 40X. B. Histological peritoneal implant: bizarre cells are present as well as in the primary tumor. Tumor cells exhibit a broad and clear cytoplasm. HE 40X.

predominantly in the pelvic region (Fig. 2) mimicking dissemination of ovarian cancer. A histological confirmation of metastatic HCC was made (Fig. 3B). The patient began palliative therapy with sorafenib. She died at 6 months due to untreatable tumor progression.

\section{DISCUSSION}

Hemoperitoneum secondary to spontaneous rupture of HCC is a relatively frequent tumor presentation in places with a high incidence of this cancer. By contrast, this is exceptional in Western countries, where most cases arise on liver cirrhosis (1). The pathophysiological mechanism is unknown (2). Its prevalence ranges from 3-15\% (3) and it should be considered in the differential diagnosis of acute hemoperitoneum. Intraperitoneal metastases are rare but their risk is elevated in tumor rupture. If the peritoneal implant is unique, which is the most common form of presentation, the treatment of choice is resection $(4,5)$. The singularity of the case described here is the debut as acute hemoperitoneum together with multiple peritoneal dissemination. Both events are exceptional, with very few cases reported in the literature (3-5).

\section{REFERENCES}

1. Fernández-Ruiz M, Guerra-Vales JM, Llenas-García J, Delgado-García JM, Gómez-Pellico C, González-Barber A. Hemoperitoneum as presentation of hepatocellular carcinoma: Experience in three cases with spontaneous tumoral rupture and review of the literature. An Med Interna 2008;25:81-4.

2. Tarantino L, Sordelli I, Calise F, Ripa C, Perrotta M, Sperlongano P. Prognosis of patients with spontaneous rupture of hepatocellular carcinoma in cirrhosis. Updates Surg 2011;63:25-30.

3. Yunoki Y, Takeuchi H, Makino Y, Murakami I, Yasui Y, Tanakaya K, et al. Intraperitoneal seeding of ruptured hepatocellular carcinoma: Case report Abdom Imaging 1999;24:398-400.

4. Ryu JK, Lee SB, Kim KH, Yoh KT. Surgical treatment in a patient with multiple implanted intraperitoneal metastases after resection of ruptured large hepatocellular carcinoma. Hepatogastroenterology 2004;51:239-42.

5. Hung MC, Wu HS, Lee YT, Hsu CH, Chou DA, Huang MH. Intraperitoneal metastasis of hepatocellular carcinoma after spontaneous rupture: A case report. World J Gastroenterol 2008;14: 3927-31. 